\title{
A new tool in the medical bag shows promise
}

\author{
Sunil Singhal, MD \\ From the Division of Thoracic Surgery, University of Pennsylvania Perelman School of Medicine, Hospital of the \\ University of Pennsylvania, Philadelphia, $\mathrm{Pa}$. \\ Disclosures: Author has nothing to disclose with regard to commercial support. \\ Received for publication Aug 7, 2015; accepted for publication Aug 8, 2015; available ahead of print Sept 3, 2015. \\ Address for reprints: Sunil Singhal, MD, Division of Thoracic Surgery, University of Pennsylvania Perelman \\ School of Medicine, Hospital of the University of Pennsylvania, Philadelphia, PA 19104 (E-mail: sunil. \\ singhal@uphs.upenn.edu). \\ J Thorac Cardiovasc Surg 2015;150:804-5 \\ $0022-5223 / \$ 36.00$ \\ Copyright $(\subset 2015$ by The American Association for Thoracic Surgery \\ http://dx.doi.org/10.1016/j.jtcvs.2015.08.020
}

In this issue of the Journal, Farjah and colleagues ${ }^{1}$ present a novel study combining 2 molecular diagnostic technologies: molecular imaging and serum molecular testing. Their group proposes that a combination of fludeoxyglucose $\mathrm{F} 18$ (INN fludeoxyglucose [18F]; FDG) positron emission tomography (PET) with a serum biomarker for vascular endothelial growth factor C (VEGF-C) is better than FDG PET alone in predicting metastatic lung cancer in hilar and mediastinal lymph nodes.

At this time, molecular imaging with FDG PET remains the primary screening device for metastatic disease to the lymph nodes, and this test remains the cornerstone of every thoracic surgeon's preoperative evaluation. This study relies on the principle that tumors have accelerated rates of glucose metabolism, leading to increased cellular uptake of the radiotracer FDG. Every surgeon knows the limitations of PET, however, including lack of sensitivity for subcentimeter lymph nodes and the absence of specific information about FDG-avid lesions.

In this deserted scene, Farjah and colleagues ${ }^{1}$ propose a refreshing new player, based on a serum biomarker, to augment the thoracic surgeon's armamentarium in preoperative evaluations. As the biology of lung cancer continues to unravel during our generation, several biomarkers have surfaced that hold significant promise. These biomarkers appear in the systemic circulation as a result of constant tumor growth and death that allows lung cancers to release considerable quantities of breakdown products into the bloodstream. Molecular diagnostics such as serum biomarkers are not new to surgical oncologists. Gynecologic oncologists routinely use cancer antigen 125 , urologist have prostatespecific antigen, pancreatic specialists depend on cancer antigen 19-9, endocrine surgeons check thyroglobulin levels, and colorectal experts order carcinoembryonic antigen titers. So it comes as no surprise that a biomarker may now exist for thoracic surgeons.

In this study, Farjah and colleagues ${ }^{1}$ suggest the use of VEGF-C to risk stratify patients with lung cancer who are likely to have metastatic disease in their lymph nodes. This is a reasonable hypothesis, given the underlying

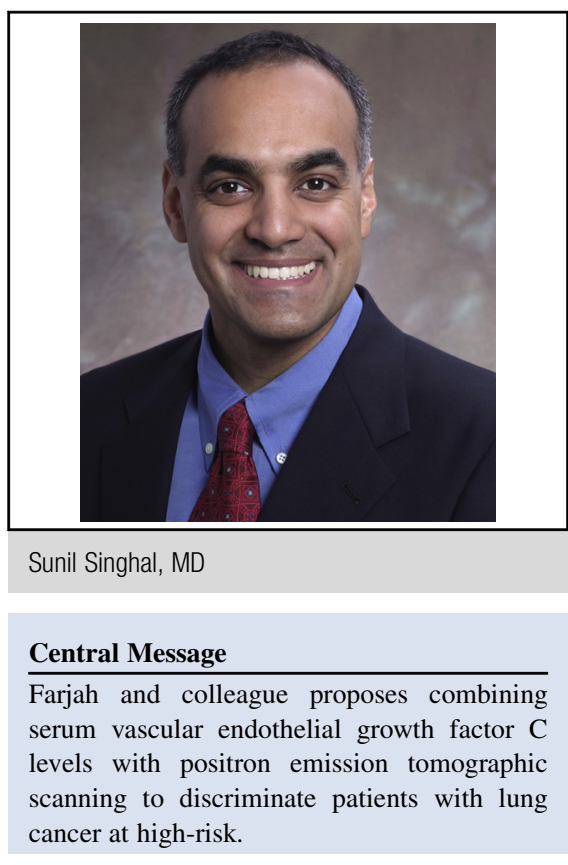

See Article page 796. premise that VEGF-C is a required growth factor in lung cancer lymphangiogenesis. Previous studies have already shown that VEGF-C plasma levels are higher in nodepositive non-small cell lung cancer. Farjah and colleagues' primary contribution is proposing a prediction model that combines information from PET and serum VEGF-C levels. ${ }^{1}$ They should be applauded for their efforts to bring a molecular diagnostic tool to the thoracic surgical community. It is quite likely that within a decade or less, many staging systems, including that of lung cancer, will have a component of molecular data that improves prognostication.

In the study of Farjah and colleagues, ${ }^{1} 58 \%$ of the patients had FDG uptake in the lymph nodes. In these patients, the positive predictive value was no better than that of flipping a coin $(50 \%)$, and the overall accuracy was $60 \%$. All patients underwent lymph node staging by endobronchial ultrasonography, mediastinoscopy, or intraoperative assessment at the time of pulmonary resection. In terms of pathology, $40 \%$ ultimately had nodal disease. By using a post hoc analysis, Farjah and colleagues evaluated serum levels of VEGF-C in the same cohort, and they found that VEGF-C was significantly higher in patients with node-positive cancer versus those with nodenegative cancer (1179 vs $733 \mathrm{pg} / \mathrm{mL})$. When the results 
of the PET scan were combined with the VEGF-C levels, the specificity, positive predictive value, and overall accuracy of selecting patients for invasive staging would have been significantly better. The group nicely looked at other variables such as primary tumor size, location, standardized uptake values, and computed tomographically guided lymphadenectomy. Again, they found that a model incorporating serum VEGF-C levels with these other variables still had superior ability to discriminate the patients who had nodal disease.

Farjah and colleagues ${ }^{1}$ appropriately point out that this is a model, and it needs to be tested. They acknowledge the limitations of their study, such as the small sample size $(\mathrm{n}=62)$ and retrospective nature. Because of their limited study size, they did not draw deeper conclusions about disease burden or N1 versus N2 involvement. Furthermore, the statistics are complex and difficult to follow at times. Nevertheless, the introduction of a "better" way to stage this disease is to be commended.
Currently, we are still finding incidental lymph node involvement in as many as 1 in 3 patients at the time of surgery. If there is any opportunity to add a diagnostic blood test that can be ordered along with the standard chemistry and coagulation studies, then we will come a far way in improving the care of our patients.

The future of molecular diagnostics, as always, will pivot on one item: assay validation. This article sets the stage and proposes a hypothesis that needs to be tested in a prospective, multicenter trial. The typical challenges, such as standardization of assays, consistent analysis of PET images, and cost-benefit analysis, will need to be sorted out. At the end of this task, however, thoracic surgeons may finally have their first reliable serum biomarker.

\section{Reference}

1. Farjah F, Madtes DK, Wood DE, Flum DR, Zadworny ME, Waworuntu R, et al. Vascular endothelial growth factor $\mathrm{C}$ complements the ability of positron emission tomography to predict nodal disease in lung cancer. J Thorac Cardiovasc Surg. 2015;150:796-803.e2 